\title{
Frequency of Unnecessarily Biopsies among Patients with Suspicion of Prostate Cancer in Syrian Men
}

\author{
Dala-Maria Bachour*, Emil Chahin, Sahar Al-Fahoum
}

\begin{abstract}
Background: The prevalence of prostate cancer is considered high in many countries, and screening tests are very important in order to detect prostate cancer in its early stages; however false positivity with these screening tests means that a lot of patients undergo unnecessary biopsy, which is an invasive procedure, for the confirmatory test. The purpose of this study was to estimate the frequency of unnecessary biopsy cases in patients referred for prostate biopsy in one of the most important and overload cancer centers in Syria. Materials and Methods: Retrospective data for a period of four years between January 2009 and December 2012 were collected in AlBayrouni University Medical hospital in Damascus, Syria. The patients from whom data were collected were referred to our histopathological department because of elevated prostate specific antigen (PSA) serum or an abnormal digital rectal examination (DRE). All patients underwent prostatic TRUS-guided biopsies. Diagnosis of prostate cancer (PCa) or benign prostatic hyperplasia (BPH) was based on histopathological examination and prostate cancers cases were graded and scored according to the Gleason score system. Results: For the 406 patients referred to biopsy, the mean \pm SD age was $58.4 \pm 23.3$ years. The mean \pm SD PSA level was $49.2 \pm 21.5 \mathrm{ng} /$ $\mathrm{ml}$. Of the total we found 237 patients diagnosed with PCa (58.4\%), 166 patients with BPH (40.9\%) and 3 cases were unable to be diagnosed $(\mathbf{0 . 7 \%})$ because of biopsy collection errors. Conclusions: Our study shows that a high percentage of patients are undergoing unnecessary biopsy, which suggests that the performed screening tests had a high level of false positive and may need re-evaluation.
\end{abstract}

Keywords: Prostate cancer - BPH - PSA - biopsy - Gleason score - Syria

Asian Pac J Cancer Prev, 16 (14), 5967-5970

\section{Introduction}

The prevalence of prostate cancer has been reported to be very high considered to be the second leading cause of cancer deaths among men in the USA and Europe. Its prevalence is very high with 684,400 estimated new cases and with 136,500 estimated deaths in developed countries in 2008 . In economically developing countries, it has been recorded to be 250,000 estimated new cases and 121,900 estimated deaths that put the prostate cancer in the sixth terms as prevalence and cause of cancer death in these countries (Ferlay et al., 2010; Jemal et al., 2010). Many studies show an elevated incidence in Middle East countries for prostate cancer and that incidence rates are rising, like the high incidence in Turkey, Jordan, Lebanon and Syria (salim et al., 2009; Ismail et al., 2013; Shamseddin et al., 2014; Zorlu et al., 2014). And that incidence is rising even in immigrating population of these countries (Nasseri et al., 2007).

Many factors may play an important role in this incidence variation, like the oldest population and the higher age of life expectancy in the western world, ethnicity, the differences in genetic, environmental and social factors like diet, sexual activity and other factors; We may also take into consideration the poor knowledge of people concerning prostate cancer screening practices and the intensity of cancer detection (Boyle et al., 2003; Nelson et al., 2003; Arafa et al., 2012; Yang et al., 2013).

Unlike most cancers, the incidence has been increasing, but the survival rate is relatively increasing too. The early detection of the disease plays an important role in diagnosis of cancer in asymptomatic patients in its beginning and applying the appropriate treatment as soon as possible (Felgueiras et al., 2014).

There are no specific symptoms associated with the beginning of prostate cancer, and the symptoms may occur in advanced or metastatic disease. Hence, the importance of developing detection tests arises in order to detect prostate cancer in its early stages in asymptomatic patients (Madu et al., 2010).

The screening tools to detect PCa include serum of PSA with or without Digital Rectal Examination (DRE). When the PSA is elevated or the DRE is abnormal we suspect $\mathrm{PCa}$ and the patient is referred to undergo biopsy, since the confirmatory test is the histopathological examination by analyzing the prostate biopsy which also 
determines the grade of the cancer by the Gleason score. The Gleason score ranges are between 2 and 10, with 2 being the least aggressive and 10 the most aggressive, and Tumors can be classified into three categories: low grade (2-3-4), intermediate grade (5-6-7) and high grade (8-910) (Humphrey, 2004; Shariat et al., 2008; Heidenreich et al., 2014).

While PSA has a high sensitivity, it has a low specificity in cancer; it is specific to the prostate gland not to the prostate cancer. Many pathological conditions like $\mathrm{BPH}$ and prostatitis could elevate the PSA serum level. The false positive results of screening test (PSA) causes a lot of unnecessary biopsy which is an invasive procedure (Burford et al., 2010).

For that, many researchers are trying to find new markers or procedures to be used in order to improve screening program tests used now, especially to counterpart the low specificity and the high number of unnecessarily biopsies (Dwivedi et al., 2011; Zheng et al., 2012; Cihan et al., 2013).

However the studies about the patients who performed unnecessarily biopsy are still very limited in our country. The purpose of this study was to estimate the frequency of unnecessarily biopsy cases in patients referred to prostate biopsy.

\section{Materials and Methods}

In this retrospective study, data were collected during 4 year between January 2009 and December 2012 in AlBayrouni University Medical hospital in Damascus, Syria. The patients from witch data was collected were referred to histopathological department from urology department because of elevated PSA serum level (cutoff for PSA serum levels $\geq 4 \mathrm{ng} / \mathrm{mL}$ ) or an abnormal DRE examination. All patients underwent prostatic TRUS guided biopsies. Diagnosis of PCa was based on histopathological examination and prostate cancers were graded and scored according to the Gleason score system. The cases were classified into two groups: BPH and PCa. Repeat biopsies for the same patient were excluded from the study, only the result for the first biopsy was included. The analysis was performed by noting down the details of the age, PSA serum levels, DRE examination results, histopathological results, and grading of cancer patients. The study protocol was approved by the Institutional Ethics Committee.

\section{Statistical analysis}

Study data were analyzed with Microsoft Office Excel 2007 and SPSS version 19 computer software. Percentages, means and ranges were given as descriptive statistical values. Values for the variables are expressed as mean \pm SD. Comparisons of variables of patients were performed using students't tests for independent samples, and a level of $\mathrm{P}<0.05$ considered as statistically significant.

\section{Results}

In this retrospective study, during the 4 year study period, 406 patients were included; all of them underwent prostatic TRUS-guided biopsies. Out of the 406 patients,
237 patients $(58.4 \%)$ had evidence of carcinoma, 166 patients $(40.9 \%)$ had $\mathrm{BPH}$ according the histological examination results, and 3 patients $(0.7 \%)$ were unable to be diagnosed because of biopsy collection errors mostly caused by small amount of biopsy sample that could not be processed. As shown in Table 1.

The ages of these patients ranged from 26 to 87 years old. The mean \pm SD age of patients was $58.4 \pm 23.3$ years, for the patients with PCa was $63.5 \pm 28.3$ years and $56.7 \pm 31.5$ years for the patients of BPH. Values did not reach statistical significance between the $\mathrm{PCa}$ and $\mathrm{BPH}$ groups $(\mathrm{P}=0.17)$.

When we distributed the study population by age groups, we noticed that the number of cancer cases was found to be highest in the older age group; because $66.6 \%$, $58,7 \%$, and $57.2 \%$ of the patients diagnosed with cancer were detected in the age range (66-90), (46-65), (25-45) respectively. As shown in Table 2.

Prostate specific antigen (PSA) values ranged from 3.2 to $504 \mathrm{ng} / \mathrm{ml}$ in patients. The mean $\pm \mathrm{SD}$ of PSA values in all patients was $49.2 \pm 21.5 \mathrm{ng} / \mathrm{ml}$, for PCa patients it was $51.2 \pm 24.6 \mathrm{ng} / \mathrm{ml}$ and it was $43.3 \pm 19.4 \mathrm{ng} / \mathrm{ml}$ in BPH patients.

In general, the levels of PSA were higher in cancer patients; however, the differences in values did not reach statistical significance between the PCa and BPH groups $(\mathrm{P}=0.08)$. Values were significantly higher in $\mathrm{PCa}$ than $\mathrm{BPH}(\mathrm{P}=0.01)$ only for the age group $(66-87)$ in this study sample.

The Gleason score (GS) values were obtained for all 237 patients diagnosed of cancer. The mean was 6. Of them $72(30.4 \%)$ patients classified in low-grade group $(\mathrm{GS}=2-3-4), 98(41.3 \%)$ patients classified in intermediate-grade group (GS=5-6-7) and 67 (28.3\%) patients classified in high-grade group (GS=8-9-10). As shown in Table 3.

Table 1. The Number and Percentage of PCa and BPH Cases Diagnosed

\begin{tabular}{lcc}
\hline & Number of cases & $(\%)$ \\
\hline PCa patients & 237 & 58.4 \\
BPH patients & 166 & 40.9 \\
Not assessed & 3 & 0.7 \\
Total & 406 & 100 \\
\hline
\end{tabular}

Table 2. Age Distribution of PCa and BPH Cases

\begin{tabular}{lcllcc}
\hline & \multicolumn{2}{c}{ PCa } & & \multicolumn{2}{c}{ BPH } \\
\cline { 2 - 3 } \cline { 5 - 6 } \cline { 5 - 6 } & Number of cases & $(\%)$ & & Number of cases & $(\%)$ \\
\hline Age groups & & & & & \\
$26-45$ & 26 & 11 & & 13 & 7.8 \\
$46-65$ & 97 & 40.9 & & 68 & 41 \\
$66-87$ & 114 & 48.1 & & 85 & 51.2 \\
\hline
\end{tabular}

Table 3. Gleason Score Distribution of PCa Cases

\begin{tabular}{lcc}
\hline & Number of cases & $(\%)$ \\
\hline Gleason score group & & \\
low-grade & 72 & 30.4 \\
intermediate-grade & 98 & 41.3 \\
high-grade & 67 & 28.3 \\
\hline
\end{tabular}




\section{Discussion}

Prostate cancer represents one of the most common cancers worldwide and an important cause of cancer mortality as well. The use of screening tests holds big significance in cancer detecting in its early stages. The benefit of the PSA test with or without DRE is well known, but the low sensitivity of these tests may lead to further diagnostic evaluation, increasing costs and use of more confirmative tests, which concerned as invasive procedure with uncomfortable aspect that physicians and patients want to avoid.

However the studies about the patients who performed unnecessarily biopsy are still very limited in our country. This study seeks to determine the frequency of PCa cases conformed by histological methods in patients referred to biopsy according to the results of screening program (the measurement of PSA with or without the DRE exam) used in Al-Bayrouni university medical hospital in Syria; taking in consideration that Al-Bayrouni University Medical hospital is the most important cancer Specialist hospital in Damascus, the capital of Syria; it receives patients from all regions of Syria, hence, the data presented here pertains to a major part of the Syrian population.

Our study demonstrated that $58.4 \%$ of patients which underwent prostatic TRUS-guided biopsies had cancer, as compared with $40.9 \%$ who had benign tumor. That suggests that a high percentage of patients referred to biopsy are found not to have cancer.

The findings of our study correlate with the finding of many other recent studies; like the study of Kash and al in Pakistan between 2012 and 2014 which shows that the cancer was detected in $50.3 \%$ of patients referred to biopsy of cause of abnormal DRE, TRUS, or elevated PSA values, in any combination (kash et al., 2014). Also the study of Mosli and al in Saudi Arabia between 2003 and 2008 demonstrated that the cancer was found only in $28.5 \%$ in patients referred to prostatic biopsy based on PSA tests or DRE results (Mosli et al., 2009). In Saudi Arabia as well, Albasri and al reported that only $17.7 \%$ of patients diagnosed with $\mathrm{PCa}$ in his study (Albasriet al., 2014).

Furthermore, the study of Rashid and al carried out in Pakistan from March 2011 to February 2012 included 203 patients with mean age $65.7 \pm 9.3$ years, also demonstrated similar results with $48.8 \%$ frequency of detection of prostate cancer cases (Rashid et al., 2013).

Likewise, in another retrospective studies in Turkey, the study of Akman and al shows that $68 \%$ of patients had $\mathrm{PCa}$ and $31 \%$ had BPH in the patients aged 75 years or older (Akman et al., 2014); And Verim and al found that from 194 men aged 75 or older also referred to biopsy for an elevated PSA or abnormal DRE, cancer was identified in 104 patients $(53.6 \%)$ (Verim et al., 2013).

Correlatively with other studies, we also notice that the diagnosis of cancer increases with increase in age; as the highest percentage $(48.1 \%)$ was found the oldest age group (66-87) years. Albasri and al reported in their study that the groups age (70-79), (80-89) and (60-69) years had the majority of PCa cases with $31.1 \%, 29.7 \%$ and $27 \%$ respectively (Albasriet al., 2014). As well known that the age is considered as a very important risk factor for Prostate cancer and incidence rates usually increase with age.

In our present study, the differences in PSA values did not reach statistical significance between the patients of prostate cancer and the patients of BPH in young men; Although that the levels of PSA were higher in cancer patients in all ages.

As shown in Table 3, the majority of patients (41.3\%) were classified in intermediate-grade cancer. The mean of Gleason Score was 6. Which correlate with other studies like the study of Anderson-Jackson and al performed in Jamaica, During six years, there were 423 patients included of which 191 (45.15\%) were diagnosed with prostate cancer, the group of moderately differentiated carcinomas (Gleason score of 6) was the largest group with 78 cases (35.29\%) (Anderson-Jackson et al., 2012). Also the study of Grivas and al in Greece found that the mean Gleason score was 6, which included 1714 patients diagnosed with prostate cancer according to the histological findings over a period of twelve year (Givas et al., 2012).

The results of the current study suggested that high frequency of unnecessarily biopsies was found. Therefore, our findings support the recommendations to re-evaluate the screening test used by adding a new marker that may support the detection capacity of the current system used especially by elevating the sensitivity.

This present study sheds light on the reliability of the screening tests used, and because of the importance of the subject, we recommend that further prospective studies should be done and also at different geographic regions which could include more factors and their impact on early prostate screening.

\section{Acknowledgements}

The authors thank for generously supplying Faculty of Pharmacy, Damascus University for funding sources.

\section{References}

Akman RY, Koseoglu H, Oguzulgen AI, et al (2014). Prostate Biopsy in the Elderly: Histologic Findings and Treatment Necessity. Asian Pac J Cancer Prev, 15, 8937-9.

Albasri A, El-Siddig A, Hussainy A, et al (2014). Histopathologic characterization of prostate diseases in Madinah, Saudi Arabia. Asian Pac J Cancer Prev, 15, 4175-9.

Anderson-Jackson L, McGrowder DA, Alexander-Lindo R (2012). Prostate specific antigen and gleason score in men with prostate cancer at a private diagnostic radiology centre in Western Jamaica. Asian Pac J Cancer Prev, 13, 1453-6.

Arafa MA, Rabah DM, Wahdan IH (2012). Awareness of general public towards cancer prostate and screening practice in arabic communities: a comparative multi-center study. Asian Pac J Cancer Prev, 13, 4321-6.

Boyle P, Severi G, Giles GG (2003). The epidemiology of prostate cancer. Urol Clin North Am, 30, 209-17.

Burford DC, Kirby M, Austoker A (2010). Prostate cancer risk management programme information for primary care; PSA testing in asymptomatic men. evidence document. NHS Cancer Screening Programmes, 143-213.

Cihan YB, Arslan A, Ergul MA (2013). Subtypes of white blood 
Dala-Maria Bachour et al

cells in patients with prostate cancer or benign prostatic hyperplasia and healthy individuals. Asian Pac J Cancer Prev, 14, 4779-83.

Dwivedi S, Goel A, Natu SM, et al (2011). Diagnostic and prognostic significance of prostate specific antigen and serum interleukin 18 and 10 in patients with locally advanced prostate cancer: a prospective study. Asian Pac J Cancer Prev, 12, 1843-8.

Felgueiras J, Silva JV, Fardilha M (2014). Prostate cancer: the need for biomarkers and new therapeutic targets. J Zhejiang Univ Sci B, 15, 16-42.

Ferlay J, Parkin DM, Steliarova-Foucher E (2010). Estimates of cancer incidence and mortality in Europe in 2008. Eur J Cancer, 46, 765-81.

Grivas N, Hastazeris K, Kafarakis V, et al (2012). Prostate cancer epidemiology in a rural area of north Western Greece. Asian Pac J Cancer Prev, 13, 999-1002.

Heidenreich A, Bastian PJ, Bellmunt J, et al (2014). EAU guidelines on prostate cancer. part 1: screening, diagnosis, and local treatment with curative intent-update 2013. Eur Urol, 65, 124-37.

Humphrey (2004). Gleason grading and prognostic factors in carcinoma of the prostate. Modern Pathology, 17, 292-306.

Ismail SI, Soubani M, Nimri JM, Al-Zeer AH (2013). Cancer incidence in Jordan from 1996 to 2009-A comprehensive study. Asian Pac J Cancer Prev, 14, 3527-34.

Jemal A, Siegel R, Xu J, et al (2010). Cancer statistics. $C A$ Cancer J Clin, 60, 277-300.

Kash DP, Lal M, Hashmi AH, et al (2014). Utility of digital rectal examination, serum prostate specific antigen, and transrectal ultrasound in the detection of prostate cancer: a developing country perspective. Asian Pac J Cancer Prev, 15, 3087-91 .

Madu CO, Lu Y (2010). Novel diagnostic biomarkers for prostate cancer. J Cancer, 6, 150-77.

Mosli HA, Abdel-Meguid TA, Al-Maghrabi JA, et al (2009). The clinicopathologic patterns of prostatic diseases and prostate cancer in Saudi patients. Saudi Med J, 30, 1439-43.

Nasseri K, Mills PK, Allan M (2007). Cancer incidence in the middle Eastern population of California, 1988-2004. Asian Pac J Cancer Prev, 8, 405-11.

Nelson WG, De Marzo AM, Isaacs WB (2003). Mechanisms of disease prostate cancer. $N$ Engl J Med, 349, 366-81.

Rashid R, Mubarak M, Kazi J (2013). Frequency of adenocarcinoma in transrectal ultrasound-guided prostate needle biopsies in men with clinical suspicion of prostate cancer and raised serum prostate specific antigen. Middle East $J$ Cancer, 4, 73-8.

Salim EI, Moore MA, Al-Lawati JA, et al (2009). Cancer epidemiology and control in the arab world-past, present and future. Asian Pac J Cancer Prev, 10, 3-16.

Shamseddine A, Saleh A, Charafeddine M, et al (2014). Cancer trends in Lebanon: a review of incidence rates for the period of 2003-2008 and projections until 2018. Popul Health Metr, 12, 4 .

Shariat SF, Roehrborn CG (2008). Using biopsy to detect prostate cancer. Rev Urol, 10, 262-80.

Verim L, Yildirim A, Basok EK, et al (2013). Impact of PSA and DRE on histologic findings at prostate biopsy in Turkish Men over 75 years of age. Asian Pac J Cancer Prev, 14, 6085-8.

Yang B, Chen WH, Wen XF, et al (2013). Role of DNA repairrelated gene polymorphisms in susceptibility to risk of prostate cancer. Asian Pac J Cancer Prev, 14, 5839-42.

Zheng X-Y, Zhang P, Xie L-P, et al (2012). Prostate specific antigen velocity (PSAV) and PSAV per initial volume (PSAVD) for early detection of prostate cancer in Chinese men. Asian Pac J Cancer Prev, 13, 5529-33.

Zorlu F, Zorlu R, Divrik RT, et al (2014). Prostate cancer incidence in Turkey: an epidemiological study. Asian Pac J Cancer Prev, 15, 9125-30. 